\title{
ESTUDO DA TAXA DE DESOXIDAÇÃO NO REFINO SECUNDÁRIO EM AÇOS DESOXIDADOS AO SILICIO E MANGANÊS
}

\author{
Thales Botelho ${ }^{*}$ \\ Gabriel Medeiros ${ }^{2}$ \\ José Adilson Castro ' \\ André Luiz Vasconcellos da Costa e Silva ${ }^{3}$
}

\section{Resumo}

O presente estudo buscou avaliar as características da desoxidação por Si-Mn em aciarias que utilizam fornos elétricos a arco (FEA). Utilizando métodos estatísticos, identificaram-se as variáveis industriais críticas de vazamento no FEA para a evolução do Si e O no início do refino no forno panela (FP). Foi empregado um modelo cinético de primeira ordem para estimar as constantes de desoxidação no início do processo no FP. Os valores obtidos experimentalmente foram $5,3 \cdot 10^{-3} \mathrm{~s}^{-1}$ e $3,6 \cdot 10^{-3} \mathrm{~s}^{-1}$. Como esperado, estes valores são superiores ao estimado para a redução do teor de oxigênio total baseado apenas na energia de agitação por injeção de gás, que foi de $1,67 \cdot 10^{-3} \mathrm{~s}^{-1}$. Isto indica que a agitação no vazamento é muito relevante na desoxidação inicial, como esperado. Foi desenvolvido um modelo que acopla a evolução da composição do aço e da escória, especialmente entre o vazamento e a chegada ao FP. O modelo confirmou a presença de uma fração sólida considerável na chegada ao FP e previu, razoavelmente, a composição da fase liquida neste instante. A atividade da sílica na escória foi mais baixa do que a esperada no equilíbrio. As limitações, potencial e aplicabilidade do modelo são discutidas, visando definir as próximas etapas para um modelo completo de adições de ferro-ligas para os aços desoxidados ao Si-Mn.

Palavras-chave: Si-Mn; Oxigênio; Desoxidação; Modelo físico; Cinética; Termodinâmica.

\section{A STUDY OF THE DEOXIDATION RATE IN SILICON MANGANESE DEOXIDIZED STEELS}

\begin{abstract}
This work has aimed at evaluating the deoxidation characteristics for Si-Mn in Electric Arc Furnace (EAF) melt shops. A statistical methodology led to the identification of the key EAF tapping process parameters that affect $\mathrm{Si}$ and $\mathrm{O}$ evolution during secondary refining. A first order kinetic equation estimated the deoxidation constants at the beginning of the ladle furnace process. The experimental values were $5.3 \cdot 10^{-3} \mathrm{~s}^{-1}$ and $3.6 \cdot 10^{-3} \mathrm{~s}^{-1}$. As expected, these values are higher than that estimated for the reduction of the total oxygen content based on the gas injection agitation energy, which was $1.67 \cdot 10^{-3} \mathrm{~s}^{-1}$, confirming the importance of the agitation caused by tapping. A model that couples slag and metal composition evolution in special from tapping to arrival at the ladle furnace (LF) was developed. The model confirmed the presence of undissolved slag solid fraction on arrival at the LF and made reasonable prediction for the liquid phase composition. The potential, limitations and applicability of each model are discussed, aiming at defining the next steps for a complete model for ferro-alloys additions to Si-Mn steels.
\end{abstract}

Keywords: Si-Mn steels; Oxygen; Deoxidation; Physical model; Kinetics; Thermodynamic

\footnotetext{
'Programa de Pós-graduação em Engenharia Metalúrgica - PPGEM, Universidade Federal Fluminense - UFF, Volta Redonda, RJ, Brasil.

${ }^{2}$ Gerdau COSIGUA, Rio de Janeiro, RJ, Brasil.

${ }^{3}$ Escola de Engenharia Industrial e Metalúrgica de Volta Redonda -EEIMVR, Universidade Federal Fluminense - UFF, Volta Redonda, RJ, Brasil
}

*Autor correspondente: thales_bot@hotmail.com 


\section{INTRODUÇÃO}

Um dos objetivos do processo de refino secundário no forno panela é ajustar a composição química do aço. Este ajuste é normalmente feito através de adições de ferro ligas. O custo de ferro ligas pode chegar a 5 a $10 \%$ do custo final do aço [I]. Por este motivo, é essencial que a quantidade de ligas e o modo de adição sejam otimizados de maneira a atingir a composição química estabelecida com o menor custo possível.

Sistemas para o cálculo de ligas e adições vêm sendo desenvolvidos há décadas, visando reduzir a dependência na experiência ou "intuição" do operador. O desenvolvimento de sistemas efetivos para este controle depende, entretanto, do conhecimento e controle das condições de vazamento das corridas no forno elétrico a arco (FEA), principalmente em relação à concentração de oxigênio solúvel no aço e a quantidade de escória que passa para a panela durante $\circ$ vazamento [2]. Oscilações significativas destes parâmetros dificultam ou inviabilizam um acerto sistemático das adições. Estas variações têm impacto direto sobre as adições no vazamento e no forno panela e, consequentemente, no controle de processo, custos de produção e acerto de composição química final [3].

Assim, o objetivo deste trabalho é desenvolver uma ferramenta, baseada em uma abordagem cinética e termodinâmica, que auxilie na correção de composição química do aço. Nesta etapa, pretendeu-se desenvolver um modelo capaz de prever o teor de oxigênio do aço e a oxidação da escória na chegada ao forno panela. Isto é, descrever os processos que ocorrem entre o vazamento da corrida do FEA e sua chegada ao forno panela, buscando evitar variações de composição química e adições de ligas incorretas no forno panela.

\section{TERMODINÂMICA E CINÉTICA DA DESOXIDAÇÃO}

A desoxidação tem como objetivo retirar parte do oxigênio solúvel contido no aço líquido. A remoção do oxigênio em solução no aço apenas pela redução da pressão parcial de oxigênio sobre o banho não é viável industrialmente [4]. Por isto, desoxidantes precisam ser empregados para remover o oxigênio do ferro.

Na produção de aços longos se utiliza, frequentemente, a desoxidação complexa [5], como por exemplo, a desoxidação com: Si-Mn, Si-Mn-Al, Ca-Si, e Ca-Si-Al.

Além do benefício da formação de óxidos mistos na desoxidação complexa, a interação metal-escória também atua de maneira a diminuir a atividade dos óxidos resultantes da desoxidação. Desta forma, a composição química da escória é tão importante quanto a adição de desoxidantes para determinar o resultado da desoxidação, isto é a composição química do aço e as inclusões formadas.

Uma parte dos óxidos da escória é resultado das reações que ocorrem no metal líquido, dentre elas a desoxidação. Em um processo conduzido adequadamente o produto da desoxidação é, em grande parte, transportado e absorvido pela escória [6].

É importante observar que o potencial químico do oxigênio no metal é afetado não apenas pelo teor de desoxidantes adicionados, mas, à medida que ocorre interação metal-escória na panela, pela atividade dos óxidos na escória. As atividades dos óxidos na escória representam a "tendência de reação química" destes óxidos [7] e, à medida que existe tempo para o metal buscar o equilíbrio com a escória, se observa que todas as reações que envolvem o oxigênio precisam buscar o equilíbrio com um único potencial de oxigênio que se estabelecerá, no aço [8].

O processo é complicado, adicionalmente, pelo fato de que a composição da escória varia significativamente na etapa imediatamente seguinte ao vazamento e até o início do processamento no FP.

Miyashita [9] utilizando o silício como desoxidante demonstrou como as concentrações de oxigênio em solução no aço e oxigênio total (oxigênio presente em inclusões ainda não removidas do metal líquido) variam em função do tempo. Mesmo com o silício, um desoxidante mais fraco que - alumínio, a reação é rápida, indicada pela rápida queda do teor de oxigênio em solução imediatamente após a adição do silício. Isto confirma que as etapas de dissolução do desoxidante, transporte e reação química entre o oxigênio dissolvido e o desoxidante e nucleação dos óxidos são muito rápidas. Por outro lado, a diminuição do teor de oxigênio total ocorre de forma mais lenta, associada aos processos de coalescimento e remoção de inclusões.

Durante o refino secundário, a agitação do aço líquido influencia no coalescimento das inclusões, no transporte dos produtos da desoxidação até a escória [7] e na homogeneização da concentração de oxigênio em solução no aço.

Recentemente, Zhang e Thomas [10] correlacionaram a potência de agitação em diferentes processos de refino secundário com o valor da constante cinética de redução do oxigênio total [7].

O resultado do estudo de Zhang e Thomas [I0] foi semelhante ao obtido por Suzuki e colaboradores [I I], em que ficou demonstrado, haver a existência de uma potência ótima de agitação [7] a partir da qual o aumento da agitação não é mais benéfico.

Este fenômeno está possivelmente associado à reoxidação que ocorre simultaneamente ao processo de remoção das inclusões, principalmente pela exposição do aço ao ar e consequente reoxidação e eventual emulsificação de escória. A reoxidação resulta, em geral, em maior teor dos óxidos menos estáveis, $\mathrm{FeO}$ e $\mathrm{MnO}$ na escória [12].

\section{MATERIAIS E MÉTODOS}

Para este estudo foram usadas corridas de aço com a composição química objetivada da Tabela I . 
O teor de oxigênio em solução foi medido antes do vazamento do FEA, na chegada ao FP e em outro momento antes de se iniciarem as adições no FP. Análises químicas do aço e da escória foram obtidas antes do vazamento no FEA, na chegada ao FP, durante e ao fim do processamento do FP.

As amostras de aço foram analisadas através do EEO (espectrômetro de emissão ótica). Os teores de oxigênio em solução foram determinados através de medidor CELOX ${ }^{\circledR}$ Heraeus Electro-Nite. As análises químicas das escórias foram feitas através de um espectrômetro de fluorescência de Raios-X.

Para os cálculos de equilíbrio metal-escória e de saturação da escória foi utilizado o software Thermo-calc e o banco de dados SLAG3.

\section{MODELAMENTO}

Formulou-se um modelo matemático visando descrever a evolução da composição química do aço e da escória na panela no período entre o vazamento e a chegada ao forno panela.

Os parâmetros discutidos em cada módulo do modelo são:

I. Módulo: Evolução da composição do aço

Tabela I. Composição química do aço (\% massa)

\begin{tabular}{cccc}
\hline Elemento & $\% \mathbf{C}$ & $\% \mathbf{S i}$ & $\% \mathbf{M n}$ \\
\hline Composição Objetivada & $0,1 \mathrm{I}$ & 0,10 & 0,43 \\
\hline
\end{tabular}

- Identificação das variáveis industriais críticas para a evolução do Si e O;

- Determinação da cinética de remoção do oxigênio do aço;

- Evolução do Si e do O no metal em equilíbrio com a $\mathrm{SiO}_{2}$ na fração liquida da escória.

2. Módulo: Formação da escória

- Escória passante: quantidade e composição;

- Adições de escorificantes: quantidade, composição e método de adição;

- Dissolução da cal: cinética do processo;

- Absorção de $\mathrm{SiO}_{2}$ da desoxidação: cinética da eliminação da $\mathrm{SiO}_{2}$ do aço;

- Evolução da composição da escória em função do tempo.

A Figura I apresenta, esquematicamente, as principais etapas consideradas no modelo. Nos próximos itens, são descritos os aspectos considerados em cada etapa, individualmente.

\section{I. Identificação das Variáveis Industriais Críticas no Vazamento para Evolução do Si e 0}

No vazamento do FEA, diversas adições são feitas ao aço. Além disto, a composição e temperatura do aço e a quantidade e composição da escória são variáveis

\section{Evolução do Aço \\ - Massa \\ - Composiçăo \\ - Oxigênio solúvel e total}

\section{Incorporação do Si}

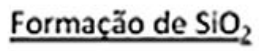
Eliminação da $\mathrm{SiO}_{2}$ formada
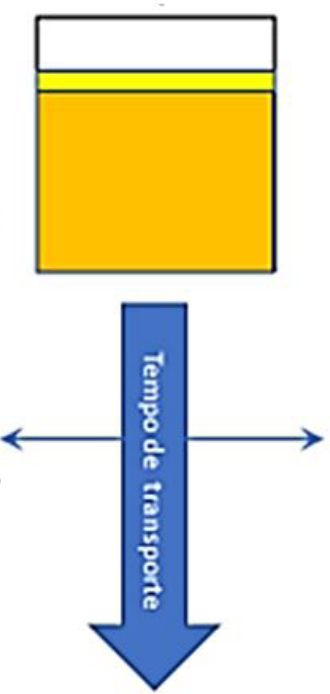

Peso e Composição do Aço e Escória na Chegada ao FP

\section{Evolução da Escória}

- Massa,

- Composição,

- Frações liquida e sólida,

- Atividade da silica,

- Solubilidade do CaO

- Redução do FeO e MnO

Incorporação de $\mathrm{SiO}_{2}$ produto da desoxidação

Dissolução da CaO

Conversão do $\mathrm{CaC}_{2}$

Figura I. Principais etapas consideradas no modelo. 
relevantes. Através de um estudo estatístico (DOE) buscou-se identificar quais seriam as variáveis críticas de vazamento para a evolução dos teores de $\mathrm{Si}$ e $\mathrm{O}$ no aço, além das quantidades e composições do aço, da escória passante e das adições realizadas.

As variáveis identificadas para o estudo estatístico foram aquelas em que havia possibilidade de serem testadas nos níveis extremos de processo sem colocar em risco a qualidade e a segurança operacional. Desta forma, foram testadas três variáveis: a temperatura de vazamento, o nível de oxidação do aço e o modo de adição do $\mathrm{CaC}_{2}$.

A Tabela 2 mostra as variáveis de entrada e o nível em que foram testadas através de um planejamento fatorial de dois níveis e com duas replicações a fim de que pudesse ser avaliada a influência de cada variável sobre a atividade do oxigênio no aço e o rendimento de Si no processo, desde o vazamento até a chegada ao FP.

A escória é desoxidada com $\mathrm{CaC}_{2}$. Foram avaliados dois modos de adição do $\mathrm{CaC}_{2}$. $\mathrm{O}$ primeiro foi a adição junto com as ligas de vazamento: a adição, portanto, foi feita diretamente no aço. $O$ segundo foi ao final do vazamento com a adição feita sobre a escória.

A análise estatística, resumida na Tabela 3 , indica que apenas o grau de oxidação no vazamento é relevante estatisticamente para atividade de oxigênio na chegada ao forno panela. Esta foi a única variável que apresentou um valor de P-valor inferior a 0,05.

Os resultados confirmam que $\circ \mathrm{CaC}_{2}$ em pó só influencia a desoxidação da escória. No período entre vazamento e chegada ao FP sua atuação é, portanto, reduzir os teores de $\mathrm{FeO}$ e $\mathrm{MnO}$, não tendo influência significativa, nesta etapa, sobre o rendimento do Si e oxigênio do aço. Assim, assumiu-se, no modelamento, que os teores de $\mathrm{FeO}$ e $\mathrm{MnO}$ da escória na panela são reduzidos, no vazamento, instantaneamente para $7 \%$, valor médio $\% \mathrm{MnO}+\% \mathrm{FeO}$ observado em 16 corridas.

A análise estatística confirmou a importância da medida e controle do teor de oxigênio do aço no vazamento para que um modelo possa ser bem-sucedido.

Tabela 2. Varáveis de Entrada Testadas

\begin{tabular}{lcc}
\hline \multicolumn{1}{c}{ Entradas } & Mínimo & Máximo \\
\hline Temperatura Vazamento (oC) & 1630 & 1660 \\
PPM_O Vazamento & 400 & 800 \\
Carbureto de Cálcio & Aço $(\mathrm{I})$ & Escória (0) \\
\hline
\end{tabular}

\subsection{Escória Passante}

Em trabalho anterior, foi desenvolvida metodologia para a avaliação da quantidade de escória passante [13]. Utilizou-se um balanço de massa dos elementos, considerando-se que os elementos adicionados ao volume de controle (a panela) estarão presentes de uma entre duas formas: como parte do aço líquido ou como parte da escória, na forma de óxidos (o enxofre não foi considerado no balanço) [13]. Este balanço e as medidas experimentais de composição do aço no vazamento e na saída do FP e composição da escória no FEA antes do vazamento e no final do processo no FP permitem estimar a quantidade de escória passante.

\subsection{Dissolução da Cal}

A cal adicionada foi aproximada como esferas de $30 \mathrm{~mm}$ de diâmetro. Assumiu-se que a dissolução é controlada pelo transporte de $\mathrm{CaO}$ na fase liquida da escória, conforme Equação I.

$$
\frac{\mathrm{dr}}{\mathrm{dt}}=\mathrm{k}_{\mathrm{CaO}}\left(\frac{\mathrm{CaO}-\mathrm{CaO}_{\mathrm{sat}}}{\rho_{\mathrm{CaO}}}\right)
$$

Onde $r$ é o raio da partícula, $\mathrm{t}$ é o tempo, $\mathrm{CaO} \mathrm{k}$ é a constante cinética da dissolução da cal, $\mathrm{CaO}$ concentração na escória, sat $\mathrm{CaO}$ concentração de saturação na escória e $\mathrm{CaO} \rho$ a densidade. Para o modelo de dissolução da cal, a constante cinética de dissolução do $\mathrm{CaO}$ foi baseada no estudo de Cheremisina e colaboradores [14] que estudaram a dissolução da cal em escórias de conversor a temperaturas típicas de aciaria.

Para a determinação do $\mathrm{CaO}_{\text {sat }}$, a composição da escória é estimada inicialmente como sendo a escória passante do FEA, com as seguintes alterações instantâneas, no vazamento:

- Fluorita adicionada no vazamento funde instantaneamente;

- $\mathrm{FeO}$ e $\mathrm{MnO}$ são reduzidos instantaneamente para $7 \%$ da escória, devido à ação do $\mathrm{CaC}_{2}$ adicionado no vazamento, como discutido no item 4. I, acima.

A partir do vazamento, sílica é incorporada a escória, conforme será discutido no item 4.4, a seguir. $O$ teor de $\mathrm{CaO}$ de saturação foi calculado empregando o software Thermo-calc e o banco de dados SLAG3, como mostra a Tabela 4, para algumas corridas.

Tabela 3. Análise de Regressão Múltipla

\begin{tabular}{ccccc}
\hline Variável & Coeficiente & Erro padrão & t & P \\
\hline Constante & $-232,2$ & 350,6 & $-0,66$ & 0,520 \\
Ppm O vaz & 0,10392 & 0,01668 & 6.23 & 0,000 \\
Temp vaz & 0,1676 & 0,2131 & $-0,79$ & 0,447 \\
Carbureto & $-2,992$ & 6,958 & $-0,43$ & 0,675 \\
\hline
\end{tabular}




\subsection{Determinação da Cinética de Remoção do Oxigênio e Evolução do Si no Aço}

O modelo de redução do oxigênio do aço e aumento da sílica na escória é, possivelmente, a parte mais complexa do processo a ser modelada.

Inicialmente, avaliou-se a evolução do teor de oxigênio dissolvido no aço entre o vazamento do FEA e a chegada ao FP. Observou-se, através de cálculos por termodinâmica computacional, que, para os teores de Si e Mn do aço estudado e as atividades calculadas para os óxidos na escória, a desoxidação é controlada pelo Si. A reação de desoxidação, portanto pode ser expressa pela Equação 2.

$$
\underline{\mathrm{Si}}+2 \underline{\mathrm{O}} \rightleftharpoons 2 \mathrm{SiO}_{2}
$$

Em vista da dificuldade de descrever precisamente os processos que ocorrem entre o vazamento e a chegada ao FP, supôs-se que a redução do oxigênio solúvel no aço, nesta etapa, obedeça a uma cinética global de primeira ordem [15]. Adotou-se o valor de 25 ppm para a concentração de oxigênio em solução em equilíbrio, conforme determinado por [12], para as mesmas condições de processo. Obteve-se, assim, a Equação 3.

$$
\ln \left(\frac{\mathrm{O}_{\mathrm{t}}-25}{\mathrm{O}_{0}-25}\right)=-\mathrm{k}_{\mathrm{c}} \mathrm{t}
$$

Ajustando-se a Equação 3 aos dados observa-se que o processo de redução do oxigênio, entre o vazamento e o final do processamento no FP não é descrito por uma única constante cinética. Aparentemente, é possível determinar uma constante cinética para a desoxidação entre o vazamento e a chegada ao FP e outra considerando todo o tempo, desde o vazamento até o final do processamento no FP, como mostra a Figura 2. O valor da constante cinética estimada para o processo entre vazamento do FEA e chegada ao FP (linha vermelha na Figura 2) é maior $\left(5,3 \cdot 10^{-3} s^{-1}\right)$ do que para o processo englobando $\circ$ tempo de tratamento da corrida (linha azul na Figura 2) $\left(3,6 \cdot 10^{-3} \mathrm{~s}^{-1}\right)$. Isto indica que a desoxidação é mais intensa entre o vazamento e a chegada ao forno panela, quando a maior parte das ferroligas

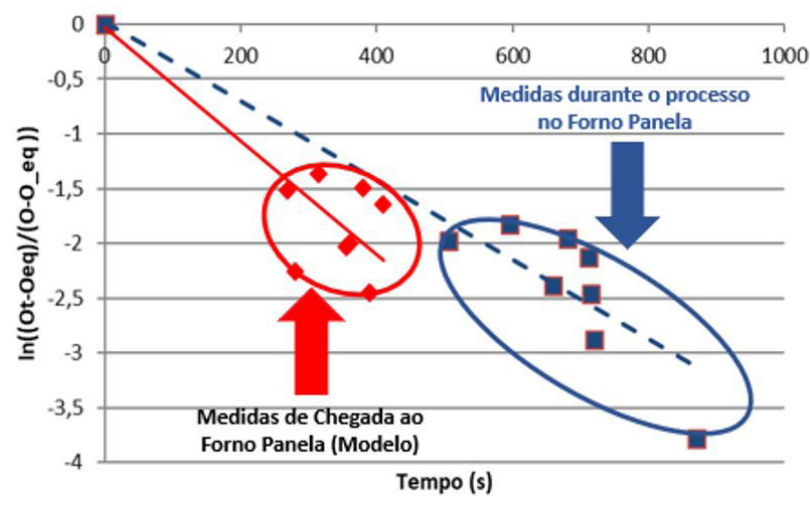

Figura 2. $\ln ((\mathrm{Ot}-\mathrm{Oeq}) /(\mathrm{Oi}-\mathrm{Oeq}))$ versus tempo. é adicionada e há intensa agitação associada ao vazamento. Quando se considera apenas o processo de desoxidação no FP, entre a chegada e saída neste equipamento, a constante cinética é de $2,22 \cdot 10^{-3} \mathrm{~s}^{-1}$

Este valor foi comparado com os dados Zang e Thomas [10] que correlacionaram a energia de agitação com a constante cinética de redução do teor de oxigênio total.

A energia de agitação do FP foi calculada usando a Equação 4:

$$
\dot{\varepsilon}=14,23\left(\frac{\dot{\mathrm{V} T}}{\mathrm{M}}\right) \log \left(\frac{1+\mathrm{H}}{1,48 \mathrm{P}_{0}}\right)
$$

Onde, $\dot{\varepsilon}$ é energia de agitação $(w / t)$, $\dot{V}$ vazão do gás $\left(\mathrm{Nm}^{3} / \mathrm{min}\right)$, $\mathrm{T}$ temperatura do banho $(\mathrm{K}), \mathrm{M}$ massa do banho $(t), H$ altura do metal líquido $(m)$, pressão do gás na superfície (atm).

Foi feita uma regressão linear entre a constante de redução do teor de oxigênio total e a energia de agitação dos dados coletados por Zhang e Thomas [10] para corridas agitadas por argônio na panela. A equação obtida está superposta aos dados de Zhang e Thomas na Figura 3. Estimou-se, para a redução do teor de oxigênio total, nas condições operacionais deste trabalho, um valor de $1,67 \cdot 10^{-3} \mathrm{~s}^{-1}$, da mesma ordem de grandeza dos valores estimados acima e comparável a constante obtida para o processo no FP.

Para uso no modelo de formação de escória e desoxidação entre vazamento e chegada ao FP, a constante cinética de redução do oxigênio em solução, determinada para esta etapa foi usada para estimar a taxa de remoção da sílica formada.

O modelo completo segue, portanto, o fluxograma apresentado na Figura 4.

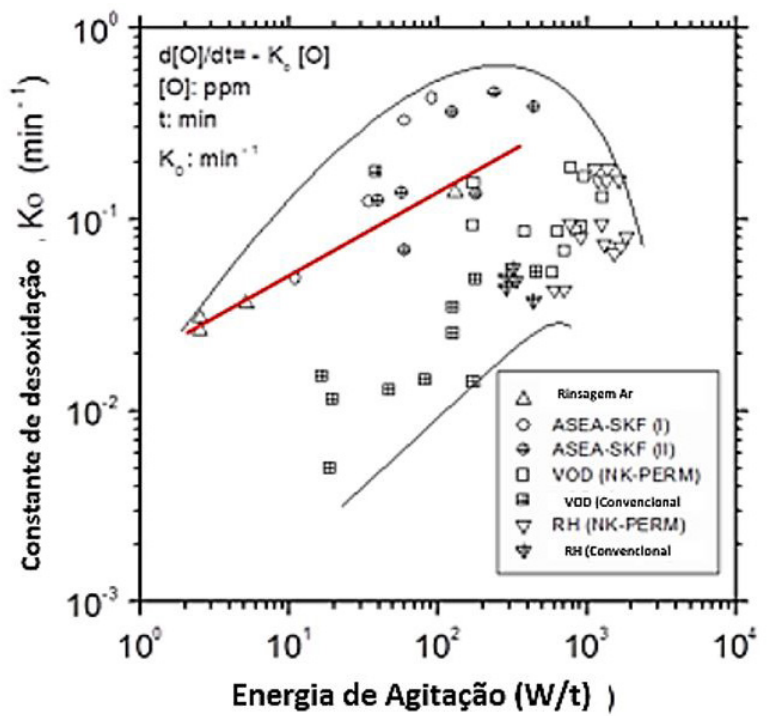

Figura 3. Efeito da agitação no grau de desoxidação [10,II]. Linha vermelha representa uma regressão linear para corridas agitadas por injeção de argônio. 


\section{RESULTADOS E DISCUSSÕES}

A evolução da desoxidação no aço e a formação da escória na panela são integradas no modelo descrito no fluxograma da Figura 4. A seguir, os resultados obtidos são comparados com alguns resultados industriais.

As Figuras 5 e 6 mostram a evolução da composição química da escória do FEA para a escória de chegada ao forno panela e a mudança de composição química estimada pelo modelo.

$\mathrm{Na}$ Figura 5 observa-se que a desoxidação da escória foi mais intensa que estimada pelo modelo. Esta diferença se deve, possivelmente, a discrepâncias entre o peso real e o estimado de escória passante conforme Tabela 4. Ainda assim, a estimativa da composição da escória de chegada ao FP é bastante razoável.

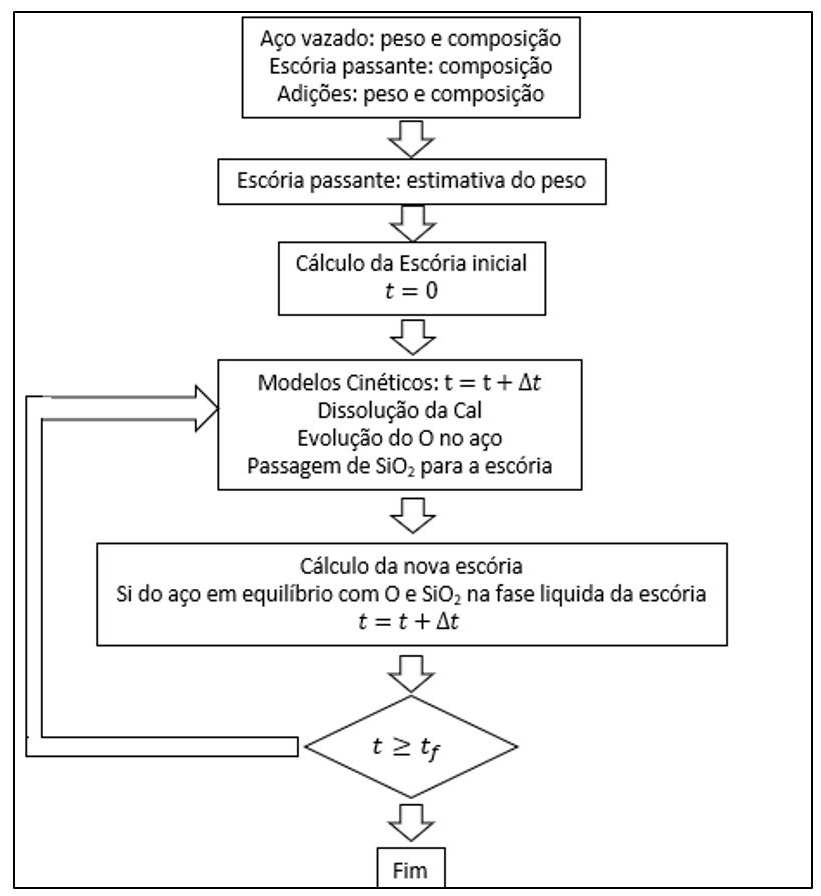

Figura 4. Fluxograma do modelo.

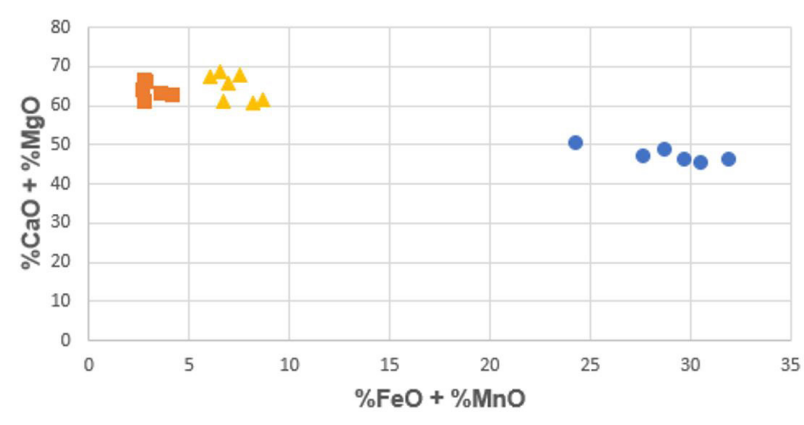

-Esc FEA 四Esc. Chegada Fp $\triangle$ Estim. Esc Chegada FP

Figura 5. Evolução do teor de $\% \mathrm{CaO}+\% \mathrm{MgO}$ e $\% \mathrm{FeO}+\% \mathrm{MnO}$ da escória Valores medidos (Esc FEA e Esc Chegada FP) e valores estimados pelo modelo (Estim. Esc Chegada FP).
A Figura 6 relaciona o nível de desoxidação da escória com os teores de $\% \mathrm{SiO}_{2}+\% \mathrm{Al}_{2} \mathrm{O}_{3}$. Os resultados das análises mostram que a fração de $\mathrm{SiO}_{2}+\mathrm{Al}_{2} \mathrm{O}_{3}$ da escória do FEA para a da panela aumenta, associada a diminuição do teor de $\mathrm{FeO}+\mathrm{MnO}$ da escória e a desoxidação do aço. Entretanto a fração de $\mathrm{SiO}_{2}+\mathrm{Al}_{2} \mathrm{O}_{3}$ da escória real foi superior aos valores estimados pelo modelo. Uma das possibilidades para o desvio entre o modelo e os resultados da análise de escória seria uma eventual adição ou pick-up (por exemplo, glaze ou escória no fundo da panela) que alterasse estes valores, embora no nível de acerto seja considerado bom.

A Figura 7 ilustra a evolução da fração sólida na escória representada pela fração de $\mathrm{CaO}$ não dissolvida na escória. Nota-se que nos primeiros instantes há uma dissolução expressiva e depois a dissolução é mais lenta, levando a uma estabilização. Esta tendência deve estar associada à diminuição da força motriz para a dissolução da cal (o gradiente de concentração representado pelo $(\mathrm{CaO} \%)$ da escória e ( $\mathrm{CaO} \%)$ sat stimado na Tabela 4).

Normalmente, observa-se que algumas corridas chegam ao forno panela com uma quantidade considerável de material não dissolvido na escória. Isto é um complicador para o modelamento das reações no FP.

Conforme discutido a composição da escória vai se alterando desde o vazamento do FEA até o final do

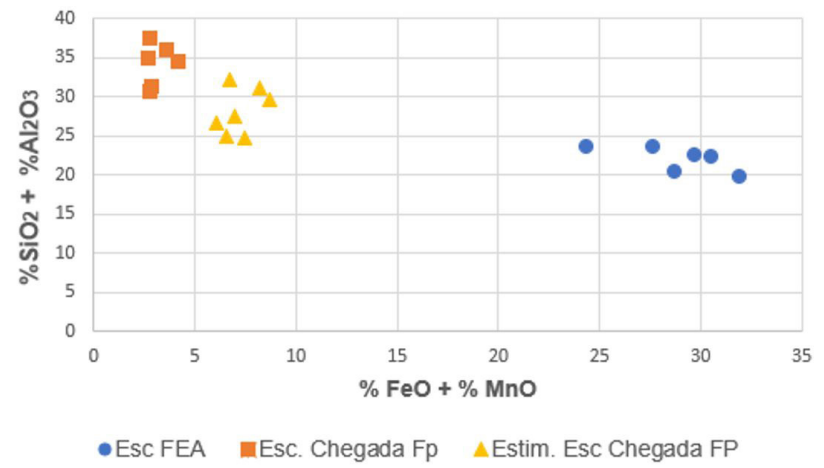

Figura 6. Evolução do teor de \%SiO2 +\%Al2O3 e \%FeO+\%MnO da escória. Valores medidos (esc FEA e Esc Chegada FP) e estimados pelo modelo (Estim. Esc Chegada FP).

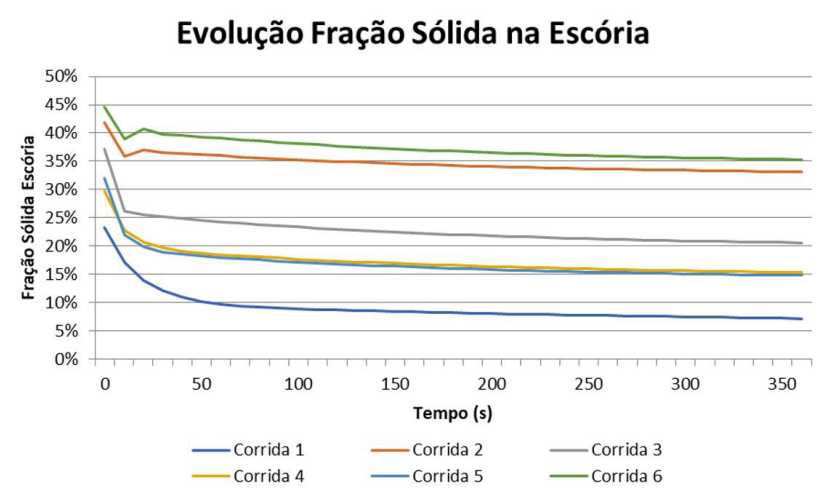

Figura 7. Evolução da Fração Sólida na Escória para diferentes corridas (Tabela 4). 
Tabela 4. Escória ao final do vazamento do FEA e o teor de $\mathrm{CaO}$ de saturação

\begin{tabular}{|c|c|c|c|c|c|c|c|c|c|}
\hline \multirow{2}{*}{ Corrida } & \multirow{2}{*}{ Temp Cheg Fp } & \multicolumn{7}{|c|}{ Estimativas de escória Passante por Balanço de Massa (Kg) } & \multirow{2}{*}{$\begin{array}{l}\mathrm{CaO} \text { saturação } \\
\text { via Thermo-calc }\end{array}$} \\
\hline & & Escória & $\mathrm{CaO}$ & $\mathrm{SiO2}$ & MgO & $\mathrm{FeO}+\mathrm{MnO}$ & $\mathrm{Al2O3}$ & CaF2 & \\
\hline I & 1613 & FEA & 705 & 297 & 220 & 140 & 100 & 166 & $51,7 \%$ \\
\hline 2 & 1595 & FEA & 257 & 102 & 63 & 55 & 47 & 166 & $41,5 \%$ \\
\hline 3 & 1570 & FEA & 314 & 128 & 123 & 58 & 37 & 166 & $46,8 \%$ \\
\hline 4 & 1630 & FEA & 471 & 208 & 164 & 92 & 59 & 166 & $47,5 \%$ \\
\hline 5 & 1604 & FEA & 377 & 180 & 207 & 81 & 52 & 166 & $45,8 \%$ \\
\hline 6 & 1576 & FEA & 227 & 86 & 61 & 41 & 29 & 166 & $40,5 \%$ \\
\hline
\end{tabular}

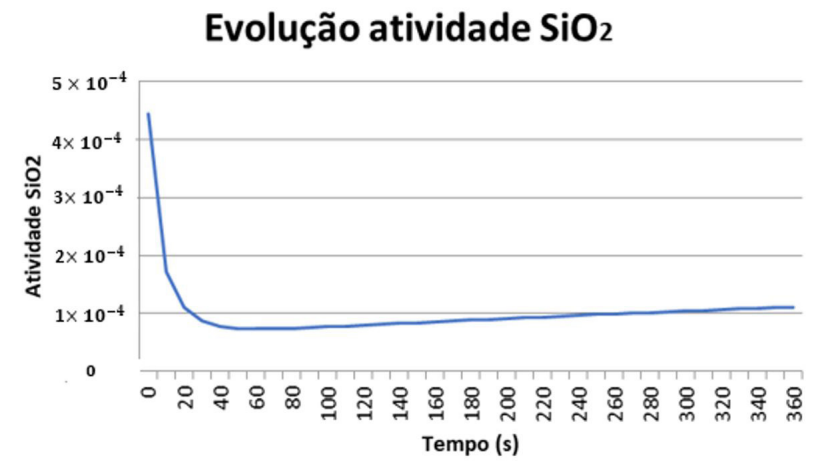

Figura 8. Variação calculada da atividade da sílica desde o vazamento do FEA até a chegada ao forno panela na corrida I (Tabela 4).

processo de refino secundário, idealmente aproximando-se do equilíbrio.

A Figura 8 ilustra a variação da atividade da sílica desde o vazamento até a chegada ao forno panela. O cálculo foi feito utilizando termodinâmica computacional a partir da variação da composição química da escória, representada pela dissolução do $\mathrm{CaO}$ e pela remoção da sílica resultante da reação de desoxidação do aço e energia de agitação. Observa-se que a atividade da sílica calculada é bastante baixa desde o momento do vazamento até a chegada ao forno panela.

Inicialmente, esperava-se que a atividade da sílica na escória evoluísse mais lentamente no processo, de modo que fosse possível estimar a concentração de oxigênio solúvel, através do equilíbrio com o silício no aço. Entretanto, este cálculo leva a valores mais baixos do que os observados de oxigênio solúvel.

A atividade da sílica mais baixa é resultado de um intenso processo de desoxidação da escória, bem como pela elevada basicidade. Entretanto, o que se propõe é que o equilíbrio com $\mathrm{Si}$ e $\mathrm{O}$ dissolvidos na fase metal líquido só ocorre plenamente na interface. Além disso, há uma importante fonte de oxigênio adicional oriunda da atmosfera e dos óxidos menos estáveis da escória ( $\mathrm{FeO}$ e $\mathrm{MnO})$, promovendo um processo secundário de reoxidação.

\section{CONCLUSÕES}

O modelo proposto utilizando dados industriais foi capaz de prever com certa precisão a composição química da escória na chegada ao forno panela, mostrando que a modelagem proposta pode ser utilizada como ponto de partida para modelos mais sofisticados capazes de descrever a desoxidação de forma mais precisa, como o modelo de fluxos acoplados [16].

Constatou-se, experimentalmente, que um modelo de primeira ordem é uma estimativa razoável para a cinética de redução do teor de oxigênio em solução na panela, do vazamento até a chegada ao forno panela. Nesta aproximação, as quedas de oxigênio solúvel e do oxigênio total foram consideradas dependentes da energia de agitação.

Os cálculos do modelo de escória mostraram que as atividades da sílica calculadas, tiveram valores abaixo do que se esperava se existisse equilíbrio termodinâmico entre $\mathrm{Si}, \mathrm{O}$ e $\mathrm{SiO}_{2}$, isto indica que o processo, mesmo durante o tratamento no FP, não atinge o equilíbrio metal escória, havendo um estado quase-estacionário entre desoxidação pelo silício e reoxidação através do "olho" aberto pela agitação ou pela redução de óxidos menos estáveis da escória ( $\mathrm{FeO}$ e $\mathrm{MnO})$.

Os resultados de aplicação do modelo indicam uma desoxidação mais alta do que a realidade. Isto indica que, possivelmente, a reoxidação do aço (pelo ar e pelos óxidos menos estáveis) deve ser incluída no modelo. A alternativa seria utilizar fatores empíricos que ajustassem as concentrações estimadas aos valores medidos

\section{Agradecimentos}

Os autores agradecem à Gerdau e ao CNPq por terem possibilitado a execução deste trabalho pela concessão da bolsa através do programa Inova Talentos de 2017 processo: 350092/2017-6.

\section{REFERÊNCIAS}

I Das AK, Mukherjee T. Optimisation of ferroalloy usage in steelmaking. Jameshdpur: NML; 1997 [acesso em 20 nov. 20I7]. Disponível em: http://eprints.nmlindia.org/2740/I/I-I3.PDF 
Botelho et al.

2 Kothari A, Ranjan R, Singh R, Kumar G, Kumar A, Agrawal A. A real-time ferroalloy model for the optimum ladle furnace treatment during the secondary steelmaking. Journal Ironmaking \& Steelmaking: Processes, Products and Applications. 20 I 7;46(3):2 I I-220. http://dx.doi.org/I0.1080/03019233.20 I 7.1368952.

3 Botelho T, Medeiros GE, Costa e Silva A, Castro JA. Estudo da taxa de desoxidação em aços desoxidados ao silicio e manganês. In: Associação Brasileira de Metalurgia, Materiais e Mineração. Proceedings of the 49th Steelmaking Seminar - International; 2018 Outubro 2-4; São Paulo, Brasil. São Paulo: ABM; 20I8. p. 775-786.

4 Costa e Silva A. Refino dos aços [acesso em 20 mar. 2019]. Disponível em: https://equilibriumtrix.net/refino/ capitulos_acs_refino/index.html

5 Graham K. Integrated ladle metallurgy control [thesis]. Hamilton: McMaster University; 2009.

6 Costa e Silva A. Non-metallic inclusions in steels - origin and control. Journal of Materials Research and Technology. 2018;7(3):283-299.

7 Costa e Silva A. Cálculos de equilíbrio em aciaria através da termodinâmica computacional. Tecnologia em Metalurgia e Materiais. 2006;3(I):45-52.

8 Costa e Silva A, Silva V, Leitão L Jr. Santos EA, Silva ACS. Avaliação do equilibrio metal-escória em metalurgia secundária. In: Associação Brasileira de Metalurgia, Materiais e Mineração. Proceedings of the 49th Steelmaking Seminar - International; 2018 Outubro 2-4; São Paulo, Brasil. São Paulo: ABM; 2018. p. 690-70I.

9 Miyashita Y. Change of the dissolved oxygen content in the process of silicon deoxidation. Tetsu-to-Hagane. 1966;52:1049-1057.

10 Zhang L, Thomas BG. State of the art in evaluation and control of steel cleanliness. ISIJ International. 2003 [acesso em 2018 jun 3];43(3):27I-29I. Disponível em: https://www.jstage.jst.go.jp/article/ isijinternational I989/43/3/43_3_27I/_article

I I Suzuki K, Kitamura K, Takenouchi T, Funazaki M, Iwanami Y. Manufacturing of high-purity steels by ladle refining. Ironmaking \& Steelmaking. 1982;9:33-37.

I 2 Leitão L Jr. Análise da reoxidação na aciaria de aços longos [trabalho de conclusão de curso]. Volta Redonda: Universidade Federal Fluminense; 2017.

I 3 Botelho T, Medeiros GE, Costa e Silva A, Serra JG. Estimativa da quantidade de escória passante no vazamento do fea através de balanço de massa. In: Associação Brasileira de Metalurgia, Materiais e Mineração. Proceedings of the 47th Steelmaking Seminar - International; 20 I6; Rio de Janeiro. São Paulo: ABM; 2016. p. 3II-3I9.

I4 Cheremisina E, Schenk J, Nocke L, Paul A, Wimmer G. Dissolution rate of pure CaO and industrial lime in converter slags. In: Associação Brasileira de Metalurgia, Materiais e Mineração. Proceedings of the 48th Steelmaking Seminar International; 2017 Outubro 2-6; São Paulo, Brasil. São Paulo: ABM; 20I7. p. 74-84.

I5 Levenspiel O. Chemical reaction engineering. 3rd ed. Nova York: John Wiley \& Sons; 1999 [acesso em 25 maio 2019]. Disponível em: https://4lfonsina.files.wordpress.com/20 I 2/I I/levenspiel-chemical-reaction-engineering.pdf

I6 Robertson D, Dei B, Ohguchi S. Multicomponent mixed-transport-control theory for kinetics of coupled slag/ metal and slag/metal/gas reactions: application to desulphurization of molten iron. Ironmaking \& Steelmaking. I 984; I I I ):4 I-55.

Recebido em: 29 Mai. 2019

Aceito em: 18 Fev. 2020 